\title{
Methyl group donors abrogate adaptive responses to dietary restriction in C. elegans
}

\author{
Maja Klapper ${ }^{1}$, Daniel Findeis ${ }^{2}$, Harald Koefeler ${ }^{3,4}$ and Frank Döring ${ }^{1 *}$
}

\begin{abstract}
Background: Almost all animals adapt to dietary restriction through alternative life history traits that affect their growth, reproduction, and survival. Economized management of fat stores is a prevalent type of such adaptations. Because one-carbon metabolism is a critical gauge of food availability, in this study, we used Caenorhabditis elegans to test whether the methyl group donor choline regulates adaptive responses to dietary restriction. We used a modest dietary restriction regimen that prolonged the fecund period without reducing the lifetime production of progeny, which is the best measure of fitness.

Results: We found that dietary supplementation with choline abrogate the dietary restriction-induced prolongation of the reproductive period as well as the accumulation and delayed depletion of large lipid droplets and whole-fat stores and increased the survival rate in the cold. By contrast, the life span-prolonging effect of dietary restriction is not affected by choline. Moreover, we found that dietary restriction led to the enlargement of lipid droplets within embryos and enhancement of the cold tolerance of the progeny of dietarily restricted mothers. Both of these transgenerational responses to maternal dietary restriction were abrogated by exposing the parental generation to choline.

Conclusions: In conclusion, supplementation with the methyl group donor choline abrogates distinct responses to dietary restriction related to reproduction, utilization of fat stored in large lipid droplets, cold tolerance, and thrifty phenotypes in C. elegans.
\end{abstract}

Keywords: Dietary restriction, Lipid droplet, Choline, Methyl group donor, Thrifty phenotype, Life history traits, C. elegans

\section{Background}

Dietary restriction (DR) induces several adaptations, including the extension of the reproductive period, prolongation of the adult life span, and the reduction of energy turnover (Fontana and Partridge 2015). Although the life span-prolonging effect of DR and the mechanisms involved have been extensively studied in a variety of organisms, the impact of DR on the regulation of lipid droplet (LD) homeostasis is limited (Baumeier et al. 2015; Bouwman et al. 2009; Miersch and Doring 2013; Palgunow et al. 2012). LDs are evolutionarily conserved fat storage organelles containing a triacylglyceride core surrounded by a phospholipid monolayer

\footnotetext{
* Correspondence: sek@molprev.uni-kiel.de

${ }^{1}$ Institute of Human Nutrition and Food Science, Molecular Prevention, Christian-Albrechts University of Kiel, Heinrich-Hecht-Platz 10, 24118 Kiel, Germany

Full list of author information is available at the end of the article
}

composed mainly of phosphatidylcholine and phosphatidylethanolamine (Zhang et al. 2010b). In addition to this classical function, LDs were recently recognized to function as storage organelles for histones during embryogenesis (Cermelli et al. 2006; Li et al. 2012), assembly platforms for specific viruses (Welte 2015), and intracellular antibacterial defense systems (Anand et al. 2012). During periods of reduced food availability and/ or growth, fatty acids are effectively delivered from LDs to maintain key energy-consuming processes (Barbosa et al. 2015; Lee et al. 2014; Narbonne and Roy 2009).

Functional genomic screens of the model organisms Caenorhabditis elegans and Drosophila melanogaster have been performed to identify genes that regulate LD formation and utilization (Ashrafi et al. 2003; Guo et al. 2008). These screens established that Arf1-COPI-mediated vesicular transport and the phosphatidylcholine synthesis pathway play important roles in the morphology and 
functionality of LDs. Many LD regulatory genes are conserved from worms to humans. Moreover, it has been shown that dietary factors, such as vaccenic acid, also regulate LD functionality (Zhang et al. 2010a). We have previously reported that in C. elegans, DR leads to the enlargement of LDs in the intestine and hypodermis (Miersch and Doring 2013; Palgunow et al. 2012), which are the main fat storage tissues of nematodes. However, little is known about the underlying cellular mechanism and the physiological role of this DR-induced LD phenotype. Because one-carbon metabolism regulates the homeostasis of phosphatidylcholine synthesis, lipogenesis, lipid droplet size, and lipolytic efficacy (Ehmke et al. 2014; $\mathrm{Li}$ et al. 2011), in this study, we used C. elegans to determine whether the methyl group donor choline plays a regulatory role in the enlargement of LDs and other adaptive phenotypes that are induced by DR.

\section{Results}

Intake of the methyl group donor choline abrogates both the enlargement of intestinal lipid droplets and the increase in the fat content of dietarily restricted worms Consistent with the results of our previous studies (Miersch and Doring 2013; Palgunow et al. 2012), DR was observed to cause enlargement of LDs in the intestine, which is one of the main fat storage tissues of $C$. elegans (Fig. 1a, a vs b). Because one-carbon metabolism plays a critical role in the regulation of both LD size and the extent of fat storage (Ehmke et al. 2014; Li et al. 2011), we supplemented the diet of the DR worms with the methyl group donor choline. We found that choline supplementation abrogates the enlargement of the intestinal LDs in DR worms (Fig. 1a, b vs d). Supplementation with the essential amino acid methionine, another methyl group donor, also reduced the size of the LDs in the intestine of DR worms (Fig. 1a, b vs f). Quantitative analysis revealed that the increased mean (Fig. 1b) and maximal (Fig. 1c) LD volume and the higher proportion of LDs $>5 \mu^{3}$ (Fig. 1d/e) were completely abrogated, or even overcompensated, in DR worms provided with supplemental choline. Choline supplementation also prevented the increase in the total LD volume (Fig. 1f) of DR worms. Because this LD parameter may serve as a proxy indicator of the extent of fat storage, we determined the triacylglyceride (TAG) contents of the worms. Consistent with the results of the LD analysis, the increase in the TAG content of DR worms was also abrogated by choline supplementation (Fig. 1g). The analysis of lipid classes revealed that choline supplementation increased the phosphatidylcholine content, which was reduced in worms subjected to DR (Fig. 1h). Taken together, our results showed that both the enlargement of intestinal lipid droplets and the increase in the fat content of dietarily restricted worms are abolished by dietary supplementation with the methyl group donor choline.

\section{Choline does not abrogate the enlargement of hypodermal lipid droplets induced by dietary restriction}

In addition to the intestine, the hypodermis functions as a major fat storage tissue in C. elegans. Confocal microscopy of the mid-segments (Fig. 1a, g-i) and heads (Fig. 2a, a-f) of the worms revealed that neither choline nor methionine supplementation prevented the enlargement of the hypodermal LDs in these regions of DR worms. Quantitative analyses of the hypodermal LDs localized in the head region of the worms showed that DR increased the mean LD volume (Fig. 2b), the number of LDs (Fig. 2c), the total LD volume (Fig. 2d)-a proxy indicator of the extent of fat storage-and the proportion of LDs $>5 \mu \mathrm{m}^{3}$ (Fig. 2e/f). None of these effects of DR was prevented by choline supplementation. Therefore, choline does not affect the DR-mediated enlargement of hypodermal LDs.

\section{Choline abrogates the delayed depletion of the fat stores of dietarily restricted worms}

Economized management of fat stores is a common adaptation to DR conditions. We therefore evaluated whether DR worms retain their fat stores more effectively than AL-fed worms (AL, ad libitum). In response to starvation ( 24 or $72 \mathrm{~h}$ ), the contents of the LDs in the mid-segment of the worms were exhausted in the AL condition (Fig. 3a, a vs c), but not in the DR condition (Fig. 3a, b vs d). Quantitative analysis revealed that the large LDs $\left(5-10 \mu^{3}\right.$, $>10 \mu \mathrm{m}^{3}$ ) of DR worms were more resistant to starvation-induced fat depletion than those of the ALfed worms (Fig. 3b-d). Next, we evaluated the fat stores of the worms by analyzing both LD volume (Fig. 3e) and lipid content (Fig. 3f). It was consistently found using both methods of evaluation that the DR worms retained approximately $60-70 \%$ of their initial fat stores in response to starvation, whereas the AL-fed worms retained approximately $40 \%$ (Fig. 3e/f) of these stores. Finally, we examined whether choline supplementation would prevent the occurrence of the fat-retaining phenotype of DR worms. We found that the decrease in LD exhaustion (Fig. 3a, b vs d) and the delay in the depletion of both the large LDs $>5 \mu \mathrm{m}^{3}$ (Fig. 3b-d) and fat stores (Fig. 3e/f) in the DR worms were abolished by choline supplementation. Taken together, our results indicate that DR worms preserve fat stores more efficiently than AL-fed animals, with the larger LDs being particularly resistant to lipolysis. These adaptive responses are abolished when the diet of DR worms is supplemented with the methyl group donor choline. 

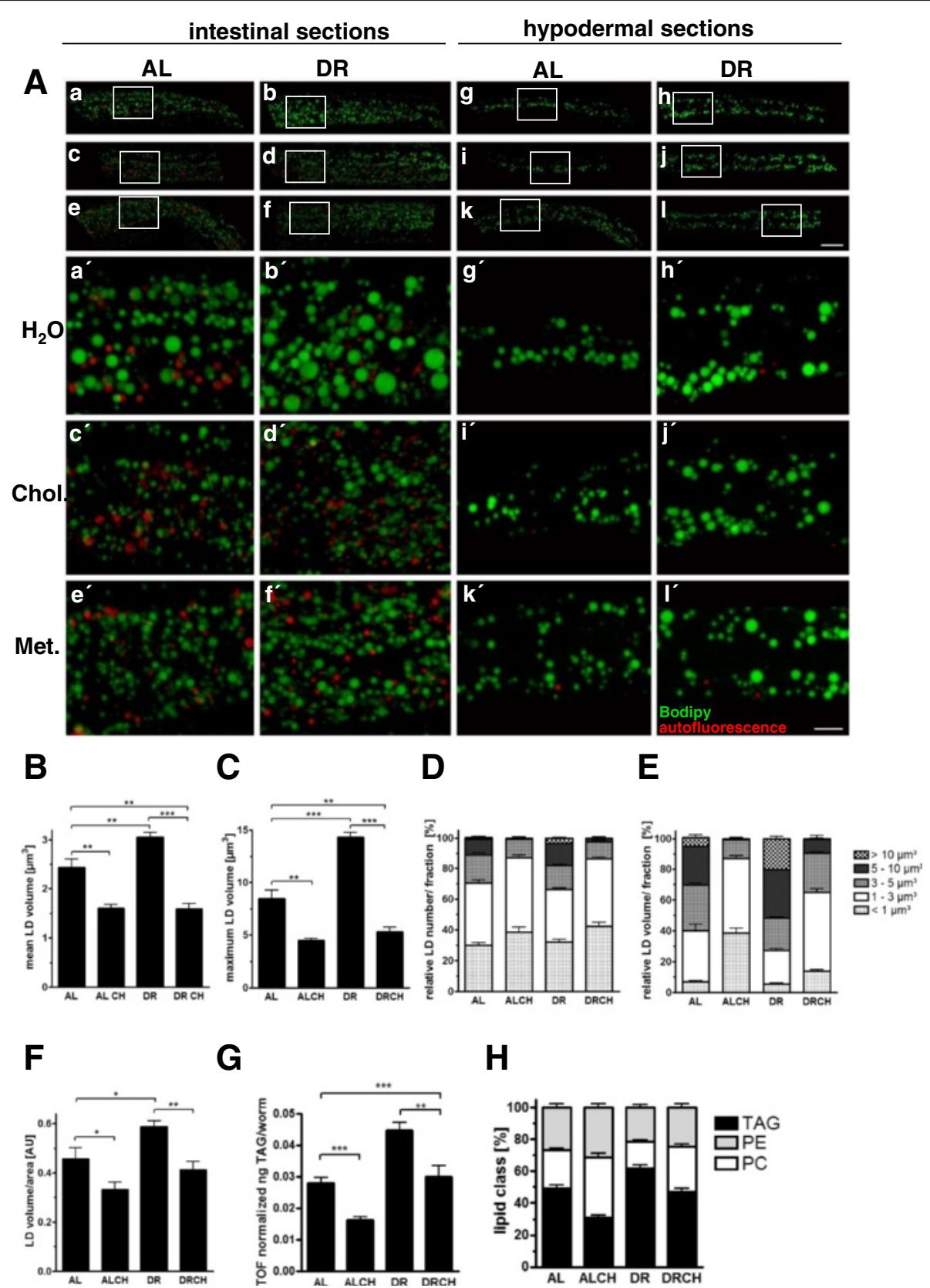

H

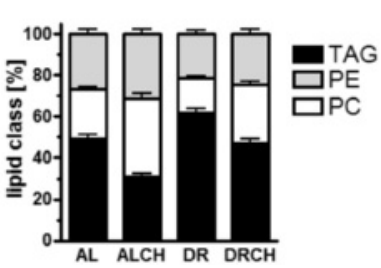

Fig. 1 (See legend on next page.) 


\section{(See figure on previous page.)}

Fig. 1 Choline and methionine supplementation abrogates the enlargement of intestinal lipid droplets and increase in fat content observed in dietarily restricted worms. a Lipid droplets in the mid-region of ad libitum-fed $L 4$ larvae $(a, c, e, g, i, k)$ and dietarily restricted $L 4$ larvae $(b, d, f, h, j, l)$, without $\left(\mathrm{H}_{2} \mathrm{O}\right.$ vehicle control; $\left.a, b, g, h\right)$ or with choline $(c, d, i, j)$ or methionine $(e, f, k, l)$ supplementation, were visualized after short-term (20 min) vital BODIPY $^{\mathrm{m}} 493 / 503$ staining using a $\times 63$ objective and Zeiss Apotome equipment. Image stacks with a depth of $10 \mu \mathrm{m}$, consisting of 21 planes at $0.5-\mu \mathrm{m}$ intervals, were captured and rendered as maximal projections ( $a-f$ ). Images $a-f$ show intestinal lipid droplets. In $g-l$, only the first six to eight images of the respective stacks are projected to demonstrate the lipid droplets in the upper hypodermal segment. The BODIPY ${ }^{\text {mim }} 493 / 503$ fluorescence signals (green) and the auto-fluorescence signals of lysosome-related organelles (red) are shown to study possible co-localizations of the signals. Scale bars, 20 and $10 \mu \mathrm{m}$ (magnifications). b-f Images of intestinal lipid droplets were analyzed using the 3D spot segmentation plug-in of ImageJ software. The mean LD volume (b), maximum LD volume (c), and total LD volume are shown. The distributions of the relative number of $L D s$ (d) and relative LD volume (e) per LD size category $\left(<1,1-3,3-5,5-10\right.$, and $>10 \mu m^{3}$ ) are shown. $\mathbf{f}$ The summarized LD volume per area (AU, arbitrary units), a proxy indicator of the extent of fat storage, is shown ( $\mathbf{f}$. The data were derived from six to nine individual worms per feeding condition in three independent experiments. $\mathbf{g}$, $\mathbf{h}$ The triacylglyceride (TAG), phosphatidylethanolamine (PE), and phosphatidylcholine (PC) contents of L4 larvae were assessed using thin-layer chromatography (TLC). g TAG content was normalized to the TOF value (time of flight), a proxy indicator of the worm's body length. Fivemicroliter samples containing lipids from equal numbers of worms (166 or 333) were dotted onto each lane to ensure that the amounts of triacylglycerides and phospholipids were in the linear range of the triolein and phospholipid standard curve, respectively. The assays were conducted in duplicate, and the results of six to eight independent experiments are summarized. AL ad libitum, ALCH ad libitum condition with choline supplementation, $D R$ dietary restriction, $D R C H$ dietary restriction condition with choline supplementation, TOF time of flight, a proxy measurement of the length of the worms

\section{Choline abrogates the enlargement of the lipid droplets of} progeny $\left(F_{1}\right)$ produced by dietarily restricted mothers $\left(P_{\mathrm{o}}\right)$ Because the increased LD size, higher fat content, and less depleted fat stores observed in DR worms are thrifty phenotypic traits, we speculated that maternal DR would affect LD size and fat-related phenotypes in the descendent $F_{1}$ progeny. Differential lipid analysis revealed similar changes in both the triacylglycerideto-phospholipid ratio and the composition of the phosphatidylcholine fatty acid species of the restricted $\mathrm{P}_{0}$ generation and the $F_{1}$ embryos ( $F_{1}$ generation) of $D R$ mothers (Additional file 1: Figure S1). These results indicate that quantitative as well as qualitative aspects of lipid metabolism are transmitted to the embryos of DR mothers. Remarkably, the $\mathrm{F}_{1}$ embryos showed enlarged vesicles localized throughout their bodies in utero (Fig. 4a, a vs b). These vesicles co-localized with the LD marker protein ATGL (Fig. 4b, a), but not with VIT-2, a yolk marker (Fig. 4b, b). The large vesicles of isolated embryos were BODIPY ${ }^{\mathrm{mt}}$ 493/503-positive (Fig. c). These results confirmed that the enlarged vesicles observed in DR-derived embryos are true LDs. A higher proportion of large LDs $>5 \mu \mathrm{m}^{3}$ were still present in DR-derived AL-fed L1/2 larvae (ALDR, 24/30) compared with AL-derived AL-fed L1/2 larvae (AL-AL, 24/30) (Fig. 4d). This persistent effect was not observed in DR-derived L4 larvae (Fig. 4d). Importantly, enlarged LDs did not occur in the embryos ( $\mathrm{F}_{1}$ generation) of $\mathrm{DR}$ mothers ( $\mathrm{P}_{0}$ generation) that were provided a diet supplemented with either choline or methionine (Fig. 4a, b vs c, d). Taken together, the results show that maternal DR causes enlargement of the LDs of offspring and that this transgenerational effect is abolished by providing methyl group donors to the mothers.
Choline abrogates the increased survival rates observed in the cold in dietarily restricted worms and in the larvae produced by dietarily restricted mothers

In nature, DR is often coincident with lower temperatures. Thus, for the ectotherm C. elegans in particular, it would be favorable for DR worms to provoke adaptations that are beneficial under cold conditions. Consistent with this theory, DR worms survived the cold for longer periods than do AL-fed worms (Fig. 5a). This DR response was also abolished by choline supplementation (Fig. 5a). Similar to the persistence of the LD phenotype, DR-derived AL-fed L2 larvae (DR-AL) (Fig. 5b), but not the respective $\mathrm{L} 4$ larvae (Fig. $5 \mathrm{c}$ ), were more resistant to cold than the AL-derived AL-fed L2 larvae (AL-AL). As observed for LD size, the transgenerational effect of DR on cold survival was abrogated by choline supplementation of the mothers' diet (Fig. 5b, DRCH-AL). Thus, both DR worms and the progeny of DR mothers show higher survival rates in the cold compared with their AL-fed counterparts. These cold-adaptive responses of DR animals are abrogated by choline supplementation.

\section{Choline abrogates the extension of the reproductive period, but not the increase in the life span of dietarily restricted worms}

The altered lipid composition, increased LD size, and higher survival rates in the cold observed in embryos derived from DR mothers might indicate that the reproduction of mothers subjected to DR is altered. As expected, DR prolonged the fecund period of the worms compared with those exposed to the AL conditions (Fig. 5d). The resulting lifetime progeny production rate, which is the best indicator of fitness, was not reduced and was even slightly increased by DR (Fig. 5e). The DRinduced extension of the reproductive period was 
A
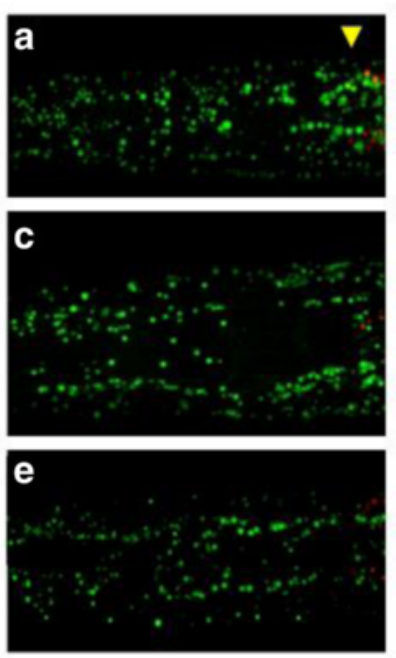

D

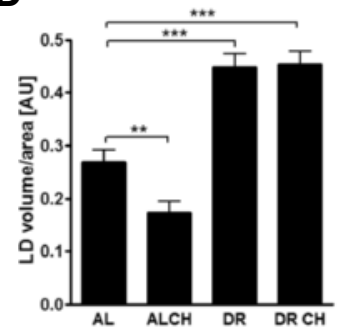

E
B

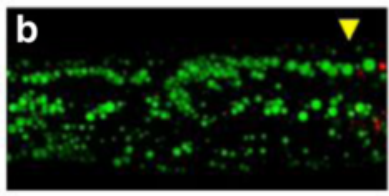

d
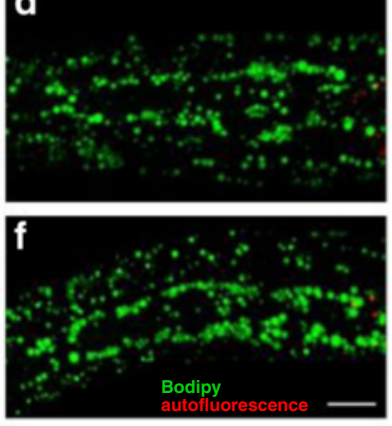

$\mathbf{F}$

C
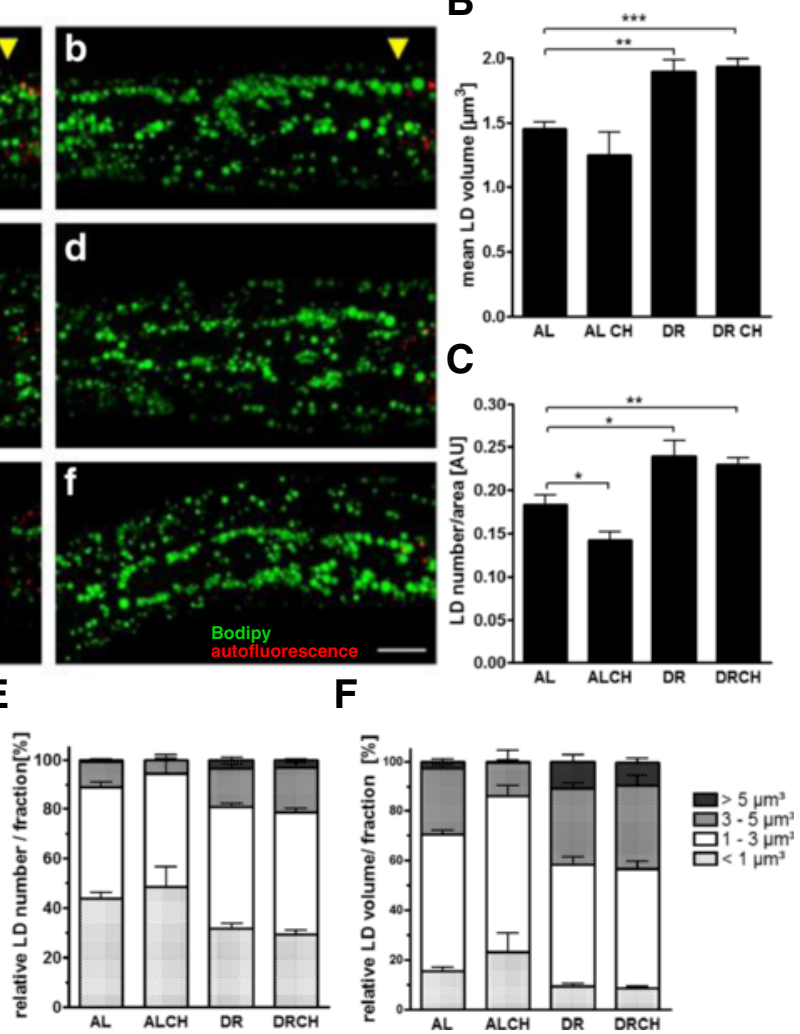

Fig. 2 Choline and methionine supplementation do not abrogate the enlargement of the hypodermal lipid droplets of dietarily restricted worms. a Hypodermal lipid droplets in the heads of ad libitum-fed $L 4$ larvae $(a, c, e)$ and dietarily restricted $L 4$ larvae $(b, d, f)$ without $\left(\mathrm{H}_{2} \mathrm{O}\right.$ vehicle control; $a, b)$ or with choline $(c, d)$ and methionine $(e, f)$ supplementation were visualized using short-term vital BODIPY ${ }^{\text {m }} 493 / 503$ staining. Image stacks with a depth of $10 \mu \mathrm{m}$, consisting of 21 focal planes at 0.5- $\mathrm{mm}$ intervals, were captured and rendered as maximum projections. Yellow arrowheads indicate the beginning of the intestine. The BODIPY ${ }^{\text {m }} 493 / 503$ fluorescence signals (green) and the auto-fluorescence signals of lysosome-related organelles (red) are shown to study possible co-localizations of the signals. Scale bars, 20 and $10 \mu \mathrm{m}$ (magnifications). b-f Quantification of hypodermal lipid droplets in the head using the 3D spot segmentation plug-in of ImageJ software. The mean LD volume (b), the summarized LD number/area (AU, arbitrary unit) (c), and the summarized LD volume/area (AU, arbitrary unit), a proxy indicator of the extent of fat storage (D), are shown. The distributions of the relative LD number $\mathbf{e}$ and LD volume $\mathbf{f}$ per LD size category $\left(<1,1-3,3-5,>5 \mu \mathrm{m}^{3}\right)$ are shown. The data were derived from six to nine individual worms per feeding condition in three independent experiments. $A L$ ad libitum, $A L C H$ ad libitum condition with choline supplementation, DR dietary restriction, DR CH dietary restriction condition with choline supplementation

completely abrogated by either choline or methionine supplementation (Fig. 5d). Surprisingly, the DR-mediated life span extension was only slightly reduced by choline or methionine supplementation (Fig. 5f), indicating that these methyl group donors exert specific effects on LD size, fat storage, survival in the cold, and reproduction.

\section{Discussion}

Almost all organisms respond to reduced food intake, or DR, via alternative life history traits that ensure survival, growth, and reproduction. In the present study, we applied a standardized DR regime (Miersch and Doring 2013; Palgunow et al. 2012) during postembryonic development. As this DR regime prevents developmental delay and growth retardation, it mimics a moderate, but sufficient food intake. The phenotypes resulting from implementing this DR regime include the accumulation of fat in large LDs, delayed depletion of fat stores, and prolongation of the fecund period without a reduction of the lifetime progeny production rate. Thus, the observed DR-induced phenotypes are highly consistent with the life history theory. Another basic theory argues that a food-restricted mother modifies the phenotype of her progeny such that they are prepared for survival in a poor nutritional environment (Barker 2001). The enlargement of the LDs and the accumulation of triacylglycerides in embryos derived from DR mothers might represent such a thrifty phenotype, assuming that the accumulated, larger embryonic LDs are more resistant to starvation-induced depletion, as observed in the mothers.

As mentioned above, our findings regarding the DRinduced phenotypes are in good agreement with the life history theory and the thrifty phenotype theory. These types of ultimate explanations are insufficient to explain 
A

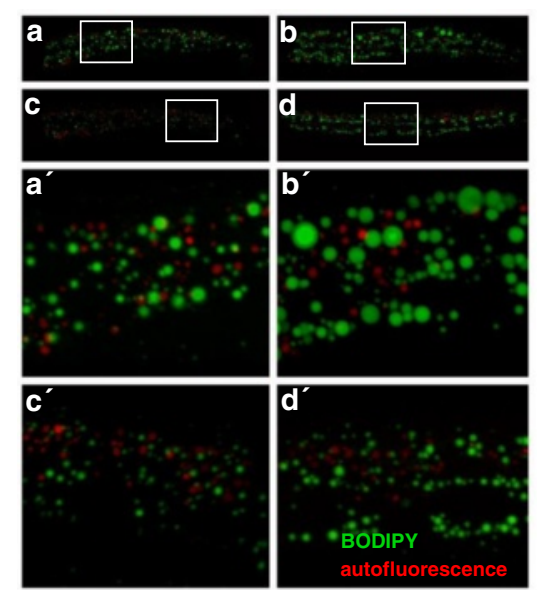

C hours $\begin{array}{lllllllll}0 & 24 & 72 & 0 & 24 & 72 & 0 & 24 & 72\end{array}$

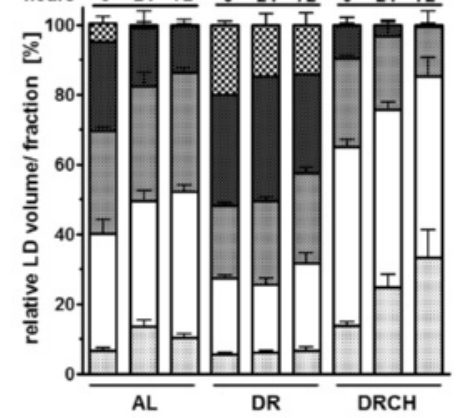

\begin{tabular}{ccc|cc|cc}
\hline D & $\begin{array}{c}\text { fraction } \\
{\left[\mu \mathrm{m}^{3}\right]}\end{array}$ & $\begin{array}{c}\text { relative LD } \\
\text { volume } \\
\% \text { of } \mathrm{T}_{0}\end{array}$ & $\begin{array}{c}\text { fraction } \\
{\left[\mu \mathrm{m}^{3}\right]}\end{array}$ & $\begin{array}{c}\text { relative LD } \\
\text { volume } \\
\% \text { of } \mathrm{T}_{0}\end{array}$ & $\begin{array}{c}\text { fraction } \\
{\left[\mu \mathrm{m}^{3}\right]}\end{array}$ & $\begin{array}{c}\text { relative LD } \\
\text { volume } \\
\% \text { of } \mathrm{T}_{0}\end{array}$ \\
\hline AL & $>10$ & 4.1 & $5-10$ & 53.5 & $>5$ & 44 \\
DR & $>10$ & 70.2 & $5-10$ & 90.1 & $>5$ & 82 \\
DRCH & $>5$ & 7.5 & $3-5$ & 54.9 & $>3$ & 41 \\
\hline
\end{tabular}

$F$ hours $\begin{array}{llllllllll}0 & 24 & 72 & 0 & 24 & 72 & 0 & 24 & 72 & \mathbf{G}\end{array}$

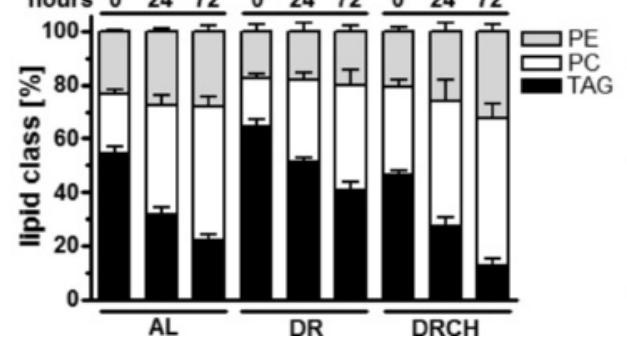

E
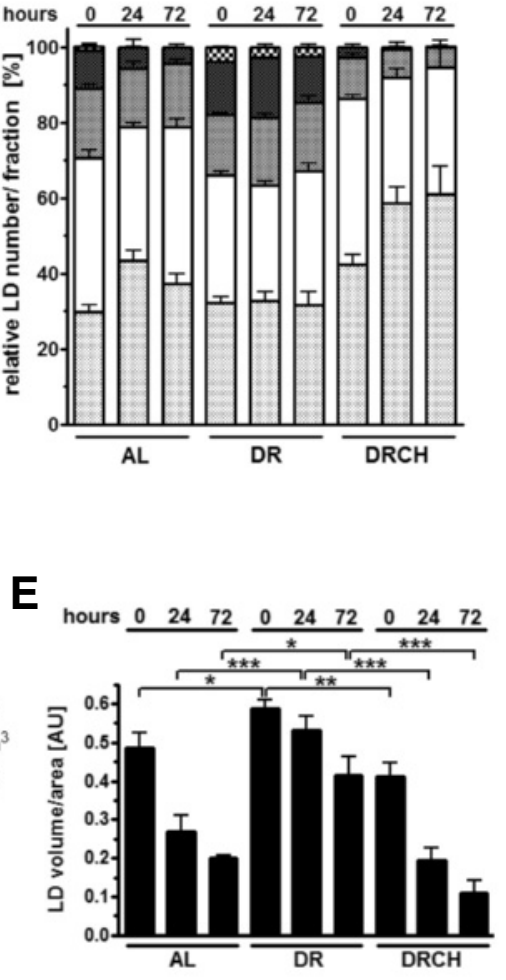

\begin{tabular}{ccc}
\hline & $\begin{array}{c}\text { TAG/PL } \\
\% \text { of } \mathrm{T}_{0}\end{array}$ & $\begin{array}{c}\text { LD volume/area } \\
\% \text { of } \mathrm{T}_{0}\end{array}$ \\
\hline AL & $40.6(54 \rightarrow 22)$ & $41.2(0.48 \rightarrow 0.20)$ \\
DR & $62.5(64 \rightarrow 40)$ & $70.8(0.59 \rightarrow 0.42)$ \\
DRCH & $27.2(46 \rightarrow 13)$ & $27.0(0.41 \rightarrow 0.11)$ \\
\hline
\end{tabular}

Fig. 3 (See legend on next page.) 


\section{(See figure on previous page.)}

Fig. 3 Choline abrogates the delayed depletion of the fat stores of dietarily restricted worms. a Depletion of lipid droplets in response to starvation (24 h) in ad libitum-fed $L 4$ larvae $(a, c)$ and dietarily restricted $L 4$ larvae $(b, d)$, without $\left(\mathrm{H}_{2} \mathrm{O}\right.$ vehicle control; $\left.a, b\right)$, or with choline $(c, d)$ supplementation. Lipid droplets were visualized after short-term (20 min) vital BODIPY ${ }^{\text {mi }}$ 493/503 staining using a $\times 63$ objective and Zeiss Apotome equipment. Image stacks with a depth of $10 \mu \mathrm{m}$, consisting of 21 focal planes at $0.5-\mu \mathrm{m}$ intervals, were captured and rendered as maximum projections. The images show intestinal lipid droplets in the mid-regions of the worms. The BODIPY ${ }^{\text {m }} 493 / 503$ fluorescence signals (green) and the auto-fluorescence signals of lysosome-related organelles (red) are shown to study possible co-localizations of the signals. Scale bars, 20 and $10 \mu \mathrm{m}$ (magnifications). b-e Quantification of intestinal lipid droplets using the 3D spot segmentation plug-in of ImageJ software. The lipid droplets of ad libitum-fed (AL) and dietarily restricted (DR) L4 larvae were analyzed at time point $T_{0}$ ( 0 h, no starvation) and in response to starvation for 24 or $72 \mathrm{~h}$. The distributions of the relative lipid droplet number/fraction (b) and the relative lipid droplet volume/fraction (c) per lipid droplet size category $\left(<1,1-3,3-5,5-10,>10 \mu^{3}\right)$ are shown. Table $\mathbf{d}$ shows the relative lipid droplet volume in response to $72 \mathrm{~h}$ of starvation (\% of $\left.\mathrm{T}_{\mathrm{o}}\right)$ among different lipid droplet size categories $\left(>10,5-10,>5 \mu \mathrm{m}^{3}\right.$ ). e shows the summarized LD volume/area (AU, arbitrary unit), a proxy indicator of the extent of fat storage. The data were derived from six to nine individual worms per feeding condition in three independent experiments. $\mathbf{f}, \mathbf{g}$ Triacylglyceride (TAG), phosphatidylethanolamine (PE), and phosphatidylcholine (PC) contents were assessed using thin-layer chromatography (TLC). The lipid contents of ad libitum-fed (AL) and dietarily restricted (DR) $L 4$ larvae were analyzed at time point $T_{0}(0 \mathrm{~h}$, no starvation) and in response to starvation for 24 or $72 \mathrm{~h}$. Table $\mathbf{g}$ shows the relative ratio of the TAG and phospholipid (PL; PE+PC) contents in response to $72 \mathrm{~h}$ of starvation (\% of $\mathrm{T}_{0}$ ). These ratios correspond to the lipid droplet volume/area. Five-microliter samples containing lipids from equal numbers of worms (166 or 333) were dotted onto each lane to ensure that the amounts of triacylglycerides and phospholipids were in the linear range of the triolein and phospholipid standard curve, respectively. The assays were conducted in duplicate, and six to eight independent experiments are summarized. $A L$ ad libitum, $D R$ dietary restriction, $D R C H$ dietary restriction condition supplemented with choline

the biological functions of the phenotypes in terms of immediate physiological or environmental factors. In searching for such factors, we consider that animals with a genetic deficiency in methyl group donors phenocopies the observed DR-induced effects. For example, $C$. elegans mutants deficient in the synthesis of SAM, the main methyl group donor found in most organisms, show enlarged LDs during both the adult and embryonic stages, increased fat stores, reduced levels of phosphatidylcholine, and delayed depletion of fat stores (Ehmke et al. 2014; Hansen et al. 2005; Walker et al. 2011). Several animal- and patient-based studies have suggested that a diet deficient in methyl group donors is an important factor in the etiology of non-alcoholic fat liver (Leermakers et al. 2015). For example, a cholinedeficient diet causes hepatic fat accumulation (Rinella and Green 2004; Zeisel et al. 1991). We suggest that endogenous and exogenous methyl group donors are critical under DR conditions and that the resulting activity of one-carbon metabolism in turn regulates LD size and fat storage. This phenomenon is particularly true for $C$. elegans and other nematodes because their food source, Escerichia coli, does not contain phosphatidylcholine. Accordingly, we found that dietary supplementation with choline as well as methionine abrogates the DR-induced fat-related phenotypes. Interestingly, folic acid and vitamin $B_{12}$ are also involved in determining the activity of one-carbon metabolism. Restriction of these vitamins may be related to the fat-related phenotypes observed under DR. A study in rats has shown that vitamin $B_{12}$ deficiency causes increased fatty acid synthesis (Matlib et al. 1979). All of these findings support the view that one-carbon metabolism is a critical gauge of food availability and a key pathway involved in regulating the adaptive storage and utilization of fat.

The most abundant phospholipid in the LD monolayer is $\mathrm{PC}$, which can be synthesized from choline (via the Kennedy pathway), or via the SAM-dependent methylation of phosphoethanolamine (in nematodes and plants) or phosphatidylethanolamine (in mammals) (Brendza et al. 2007; Palavalli et al. 2006). It has been shown that depletion of PC or loss-of-function mutations of the genes that mediate methylation-dependent PC synthesis (sams-1 and pmt-1) leads to enlargement of LDs (Bartz et al. 2007; Guo et al. 2008; Szymanski et al. 2007; Walker et al. 2011). Further studies revealed that LD enlargement occurs when there is a reduced level of PC due to fusion of the existing LDs (Guo et al. 2008; Krahmer et al. 2011; Li et al. 2011; Walker et al. 2011). Based on these findings, we propose that the expansion of LDs induced by DR is caused by the decreased availability of methyl group donors and subsequent reduction of PC levels, which in turn promotes LD fusion. This hypothesis is supported by the observation that dietary supplementation with the methyl group donors choline and methionine increases the PC content and decreases LD size in DR worms.

Remarkably, embryos derived from DR mothers also exhibit a decreased PC content and very large LDs. Both of these phenotypes can be abolished by choline or methionine supplementation of the restricted diet of the mothers, indicating that these methyl group donors are provided to the embryos maternally. Consistent with this conclusion, the embryos of a methyl group donordeficient mutant (sams-1) also exhibit large LDs. As enlarged embryonic LDs as well as cold tolerance are also 
A
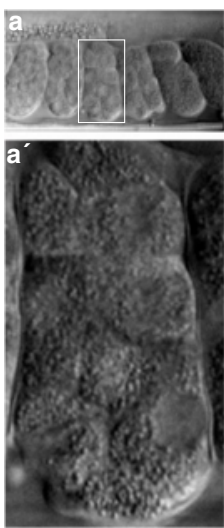

C
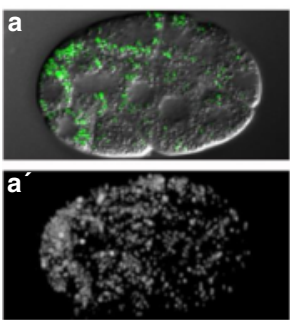
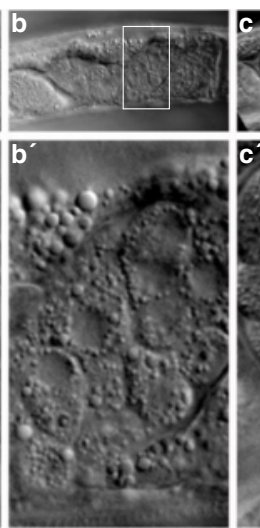
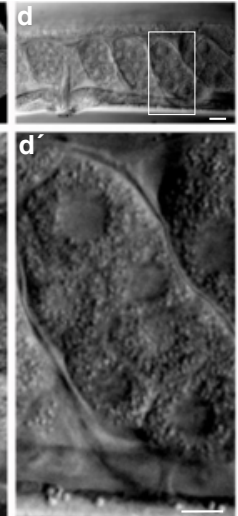

B

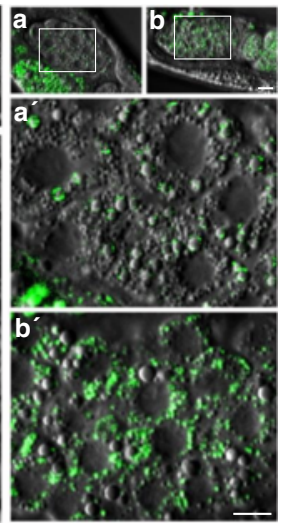

D

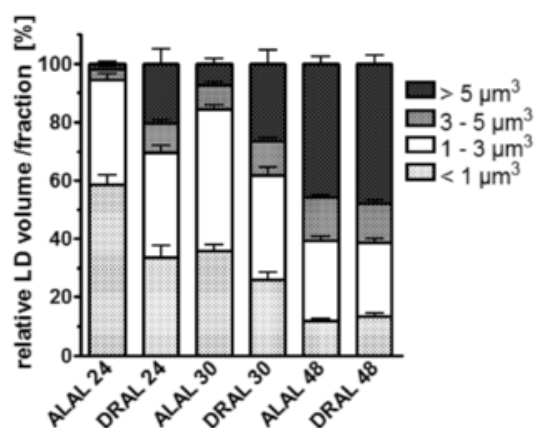

Fig. 4 Choline and methionine abrogate the enlargement of the lipid droplets of in utero embryos $\left(F_{1}\right)$ produced by dietarily restricted mothers $\left(P_{0}\right)$. a DIC images of in utero embryos ( $F_{1}$ generation) derived from ad libitum-fed $(A L, a)$ and dietarily restricted (DR, $b$ ) adult worms ( $P_{0}$ generation) supplemented with choline $(c)$ or methionine $(d)$. Images were captured using a $\times 63$ objective. Scale bar, $20 \mu m$. Enlargements of the framed areas are shown in $a^{\prime}-d^{\prime}(\times 5$, scale bar, $10 \mu \mathrm{m})$. b The lipid droplet marker ATGL::GFP $(a, a)$ and the vitellogenin marker Vit-2::GFP $(b, b)$ are shown. Images were captured using a $\times 100$ objective. Scale bar, $20 \mu \mathrm{m} ; \times 5$ magnification $a^{\prime}, b^{\prime}$, scale bar, $10 \mu \mathrm{m}$. $\mathbf{c}$ Lipid droplets from isolated embryos obtained from ad libitum-fed $(A L, a)$ and dietarily restricted $(D R, b)$ adult worms were visualized using short-term vital BODIPY ${ }^{\mathrm{TM}} 493 / 503$ staining. Merged DIC and BODIPY $^{\text {TM }} 493 / 503$ fluorescence images $(a, b)$ and fluorescence images $\left(a^{\prime}, b^{\prime}\right)$ are shown. Image stacks $\left(a^{\prime}, b\right)$ with a depth of $10 \mu$ m, consisting of 21 focal planes at $0.5 \mu \mathrm{m}$ intervals, were captured and rendered as maximum projections of the BODIPY ${ }^{\mathrm{m}} 493 / 503$ signals. $\mathbf{d}$ Quantification of intestinal lipid droplets in ad libitum-fed $(A L) L 1$ larvae ( $F_{1}$ generation; 24 h after hatching), L1/L2 larvae ( $F_{1}$ generation; 30 h after hatching), and $L 4$ ( $F_{1}$ generation; $48 \mathrm{~h}$ after hatching) obtained from ad libitum-fed (AL) and dietarily restricted (DR) adult worms ( $P_{0}$ generation). Analyses were performed using the 3D spot segmentation plug-in of ImageJ software. The distribution of the relative lipid-droplet volume/fraction ratio per lipid droplet size category $\left(<1,1-3,3-5,>5 \mu \mathrm{m}^{3}\right)$ is shown. The data were derived from six to nine individual worms per feeding condition in three independent experiments. ALAL24/30/48, ad libitum feeding of the $P_{0}$ generation and ad libitum feeding of $F_{1}$ larvae 24, 30, or 48 h after hatching; DRAL24/30/48, dietary restriction of the $P_{0}$ generation and ad libitum feeding of $F_{1}$ larvae 24,30 , or $48 \mathrm{~h}$ after hatching

observed in DR mother-derived L1/2 larvae, we suggest that the maternally provided methyl group donors persist in the progeny. Thus, the existence of a relationship between the availability of methyl group donors, PC contents, and LD size provides an explanation for the observed DR-induced fat-related phenotypes and their abolishment through choline or methionine supplementation. As the reduced PC content, increased LD size, and adaptation to cold are inherited by the next generation and can be abolished by supplementation with methyl group donors, the one-carbon metabolism of the mothers may play an important role in the occurrence of these thrifty phenotypes. Nevertheless, we cannot exclude that the inherited effect has something to do with histone methylation and subsequent gene expression. This should be studied in future experiments focusing on epigenetic effects.

We found that the starvation-induced depletion of fat stores, particularly that of the large LDs, is reduced in DR worms. This fat-sparing effect can also be abolished by supplementation with methyl group donors. In Drosophila, it has been shown that DR increases starvation resistance (Burger et al. 2007). Because the surfaceto-volume ratio is lower in larger LDs than in smaller LDs, the rate of degradation of the former by lipases might be decreased. Consistent with this hypothesis, a $C$. elegans mutant (dhs-28) deficient in peroxisomal $\beta$ oxidation is characterized by extremely large LDs that 

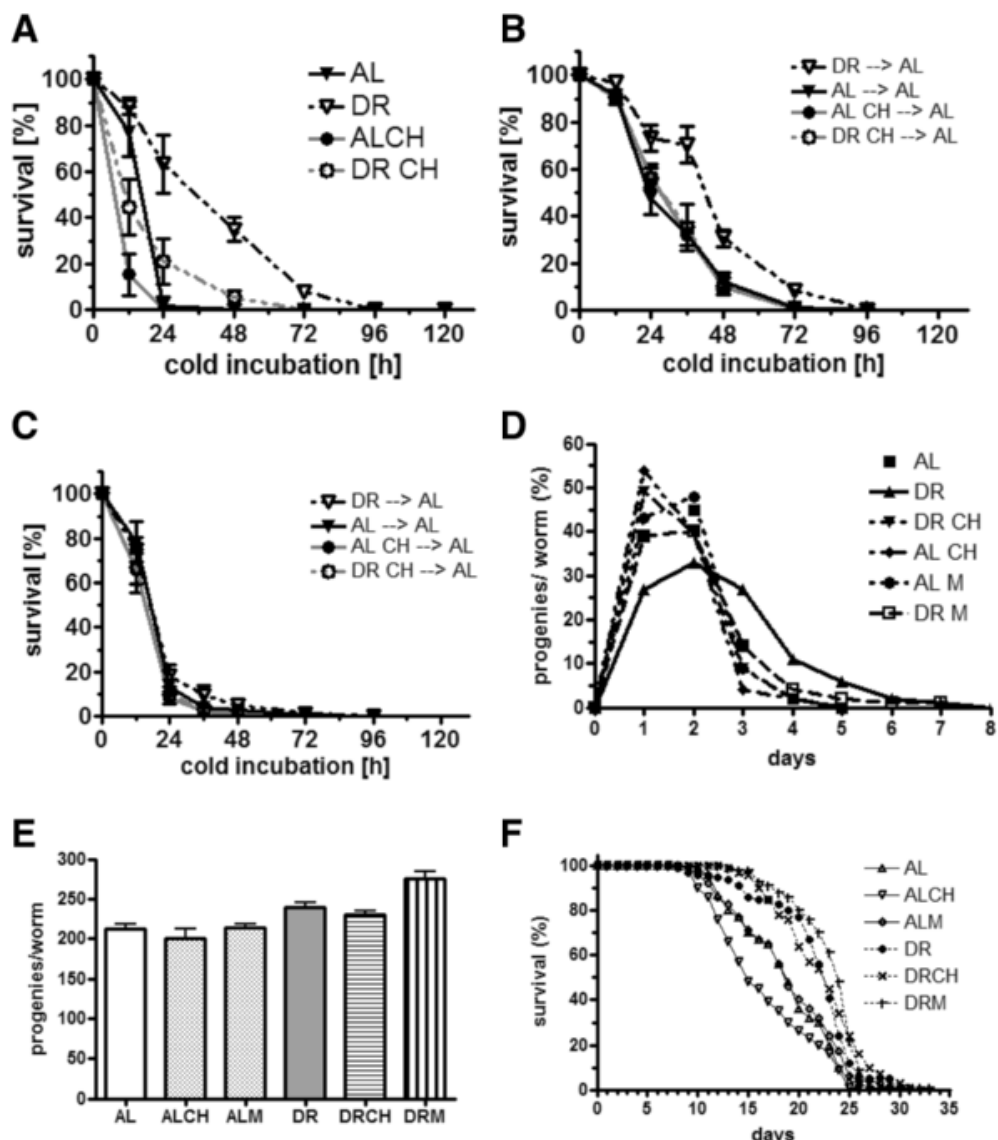

Fig. 5 Choline abrogates the extension of the reproductive period and increased survival rate in the cold in the $P_{0}$ and $F_{1}$ generations, but not the increase in the life span of dietarily restricted worms. a Survival curves of ad libitum-fed (AL) and dietarily restricted (DR) young adult worms without $\left(\mathrm{H}_{2} \mathrm{O}\right.$ vehicle control; $\mathrm{AL}$, DR) or with choline $(\mathrm{AL} C \mathrm{CH}, \mathrm{DR} \mathrm{CH})$ supplementation, following their transfer from 20 to $0{ }^{\circ} \mathrm{C}$. The data were obtained in three independent experiments. b, c Survival curves of AL-fed L1 larvae (b) or AL-fed L4 larvae (c) obtained from either AL-fed or DR adult worms, without $\left(\mathrm{H}_{2} \mathrm{O}\right.$ vehicle control; $\mathrm{AL}$, DR) or with choline $(\mathrm{AL} C \mathrm{CH}, \mathrm{DR} \mathrm{CH})$ supplementation, following their direct transfer from 20 to $0{ }^{\circ} \mathrm{C}$. The data were obtained in three independent experiments. $D R / A L \rightarrow A L, D R / A L)$ of the $P_{0}$ generation and $A L$ of $F_{1}$ larvae; $D R C H \rightarrow A L$, $D R$ with choline supplementation of the $P_{0}$ generation and $A L$ feeding of $F_{1}$ larvae. $\mathbf{d}$ - $\mathbf{f}$ Time-course of the progeny production (d), length of the reproductive period (e), and lifetime progeny production rate (f) of ad libitum-fed (AL) and dietarily restricted (DR) worms, without ( $\mathrm{H}_{2} \mathrm{O}$ vehicle control; $\mathrm{AL}$, $D R$ ) or with choline (AL CH, DR CH) or methionine (AL M, DR M) supplementation. $\mathbf{g}$ Life span curves representing the typical results of two to four independent experiments using ad libitum-fed ( $A L$ ) and dietarily restricted (DR) worms, without $\left(\mathrm{H}_{2} \mathrm{O}\right.$ vehicle control; $\mathrm{AL}$, $\left.\mathrm{DR}\right)$ or with choline (AL CH, DR CH) or methionine (AL M, DR M) supplementation

are more resistant to lipase-mediated lipolysis compared with smaller LDs (Zhang et al. 2010a). Moreover, studies in flies have shown that the large LDs that form due to reduced PC levels are more resistant to lipolysis (Guo et al. 2008; Krahmer et al. 2011). In mutant mice, the generation of small LDs leads to enhanced lipolysis (Nishino et al. 2008). The size of LDs is functionally important in the supply of fatty acids. From this point of view, a decreased rate of supply of fatty acids derived from large LDs may contribute to the prolonged reproductive period of DR worms. This hypothesis should be tested in future studies using mutants that are not responsive to starvation-induced fat depletion.

\section{Conclusions}

We have demonstrated that subjecting $C$. elegans to a moderate DR regime during the postembryonic period induces the prolongation of the reproductive period, the accumulation and delayed depletion of large LDs and whole-fat stores, an increased survival rate in the cold, the enlargement of LDs, and a higher level of cold resistance in the filial generation. Because all of these effects are prevented by dietary supplementation with methyl group donors, we conclude that one-carbon metabolism is a critical gauge of food availability, a key pathway in regulating the adaptive storage and utilization of fat, and an important determinant for the induction of thrifty phenotypes. 


\section{Methods}

\section{Worm strains, culture, dietary restriction}

The wild-type strain used in this study was Bristol N2. A VS20 strain expressing an ATGL-1::GFP fusion protein [atgl-1p::atgl-1::GFP] was used as a lipid droplet reporter strain. The strain pwls[vit-2p::vit-2::GFP] was used for the visualization of vitellogenin-containing yolk granules. The worms were cultured at $20{ }^{\circ} \mathrm{C}$ on nematode growth medium (NGM) plates using E. coli OP50 as the food source, following standard methods (Brenner 1974). For the supplementation experiments, solutions of choline (10 $\mathrm{mM})$, ethanolamine $(10 \mathrm{mM})$, betaine $(10 \mathrm{mM})$, or methionine $(1 \mathrm{mM})$ were spread on the bacterial lawn. Age synchronization of the nematodes was achieved through hypochlorite treatment of gravid adults. Dietary restriction of the worms was achieved as previously described (Palgunow et al. 2012). In short, adjusted optical densities $\left(250 \mu \mathrm{l}, \mathrm{OD}_{600} 0.7\right.$ or 1.5$)$ of the E. coli strain OP50 were spread onto antibiotic-free agar plates (without peptone) and incubated for $16 \mathrm{~h}$ at $37{ }^{\circ} \mathrm{C}$. This leads to different amounts of bacteria per agar plate depending on the OD of seeded OP50. To further standardize food availability per worm, 500 synchronized embryos were sorted onto AL and $\mathrm{dDR}$ agar plates by flow cytometry and were cultivated at $20{ }^{\circ} \mathrm{C}$ until reaching the L4 or adult stage. To exclude starvation, L4 larvae and adult worms were transferred daily to fresh agar plates.

\section{Number of progeny and reproductive period}

To determine the number of progeny, $48 \mathrm{~h}$ after synchronization and cultivation under a particular condition, L4 larvae were placed on plates containing a ring of palmitic acid to prevent their escape. Five worms per plate were cultivated for an additional $48 \mathrm{~h}$. This time point was considered day 1 of adulthood and progeny production. The animals were subsequently transferred to new plates at 24-h intervals until the end of their reproductive period. The progeny on the residual plates were counted the day after the adults were transferred. Three plates were analyzed for each condition in three independent experiments. The reproductive period was calculated as the period during which $95 \%$ of the progeny were produced.

\section{Survival rate under cold conditions}

Immediately before the worms were shifted to $0{ }^{\circ} \mathrm{C}$, the NGM plates were divided into four segments that were placed in different positions in the incubator. The nematodes were transferred to $0{ }^{\circ} \mathrm{C}$ as larvae or young adults, and survival was monitored at specific time points. The samples were analyzed in triplicate for each condition and time point. The survival rate was calculated as the percentage of dead individuals after $24 \mathrm{~h}$ of recovery at $20{ }^{\circ} \mathrm{C}$ among the total number of worms that had been counted immediately after incubation at $0{ }^{\circ} \mathrm{C}$. Worms were scored as dead if they did not respond when gently touched with a platinum wire.

\section{Starvation experiments}

Synchronized worms were grown for 48 h on NGM agar plates until reaching the L4 stage. Approximately 4000 worms were harvested from 10 to 12 NGM plates/condition and were washed three times with M9 buffer. Control worms ( $0 \mathrm{~h}$ of starvation) with or without choline supplementation were immediately sorted using a COPAS Biosort flow cytometric system into Precellys vials (1000 worms/sample) and were stored at $-80{ }^{\circ} \mathrm{C}$ until further processing. To induce starvation, worms were cultivated in 15-ml Falcon tubes in a Nutator mixer in $3 \mathrm{ml}$ of $\mathrm{M} 9$ buffer supplemented with an antibiotic (dilution: 1:100, Cell Culture Guard, AppliChem) and choline or the vehicle $\left(\mathrm{H}_{2} \mathrm{O}\right)$. After 24 and $72 \mathrm{~h}$ of starvation, the worms were collected as described above for the control worms ( $0 \mathrm{~h}$ of starvation). The samples were used for TLC and protein quantification. An aliquot of live worms grown under each condition $(0,24$, or $72 \mathrm{~h}$ of starvation) was subjected to vital BODIPY ${ }^{m+4} 493 / 503$ staining for analysis of the number and size of the lipid droplets.

\section{BODIPY $^{\text {Tm }} 493 / 503$ vital staining and fluorescence imaging}

To visualize fat storage in worms and embryos, vital BODIPY $^{\mathrm{m}} \quad 493 / 503$ (Invitrogen, Darmstadt, Germany) staining was performed as previously described (Klapper et al. 2011). Briefly, freshly harvested worms were washed three times with M9 buffer and were incubated in $500 \mu \mathrm{L}$ of BODIPY ${ }^{\mathrm{min}} 493 / 503$ solution $(6.7 \mu \mathrm{g} / \mu \mathrm{l}$ in $\mathrm{M} 9$ buffer) for $20 \mathrm{~min}$. After washing twice with M9, the worms were anesthetized using sodium azide $(1 \%)$ and immediately examined via microscopy. BODIPY ${ }^{\text {now }}$ 493/503 fluorescence was visualized using an Axio Imager system and a Z1 microscope equipped with a $38 \mathrm{HE}$ filter (excitation: BP 470/40, beam splitter: FT 495, emission: BP $525 / 50$ ), coupled to an apotome-sectioning system (ApoTome.2, Zeiss). To distinguish between BODIPY ${ }^{\text {mix }}$ 493/503 positive structures and auto-fluorescent lysosome-related organelles, auto-fluorescence was imaged using the DAPI channel. GFP fluorescence was visualized with a $38 \mathrm{HE}$ filter (excitation BP 470/40; beam splitter FT 495; emission BP 525/50).

\section{Analysis of the number and size of lipid droplets} BODIPY 493/503-stained lipid droplets localized in selected regions were imaged via $3 \mathrm{D}$ fluorescence microscopy using an Axio Imager $\mathrm{Z} 1$ microscope and a Plan-Apochromat $\times 63 / 1.3$ oil immersion objective. The apotome-sectioning system was used to collect $\mathrm{z}$-stacks with a step size of $0.5 \mu \mathrm{m}$. The z-stacks comprised 21 
planar images (format, $138.4 \times 104 \mu \mathrm{m} ; 692 \times 520$ pixels). Images were captured using an AxioCam MRm (Zeiss) system. Image software (version $1.47 \mathrm{~h}$ ) and the 3D spot segmentation plug-in were used to analyze the z-stacks. BODIPY $^{\text {mim }} 493 / 503$-positive structures were automatically identified, and the volume of each droplet was evaluated by totaling the number of voxels per droplet. If necessary, the brightness and contrast levels were manually adjusted before performing the calculations.

\section{Thin-layer chromatography}

After thawing worms stored at $-80{ }^{\circ} \mathrm{C}$, worms collected using the COPAS Biosort system were analyzed by thinlayer chromatography (Matyash et al. 2008). Lipids were extracted from 1000 or 2000 homogenized worms (Precellys 24 homogenizer; full speed; $3 \times 10$ s) using a solution containing $375 \mu \mathrm{l}$ of methanol, $1250 \mu \mathrm{l}$ of methyl-tertbutyl-ether [63], and $312 \mu \mathrm{l}$ of $\mathrm{H} 2 \mathrm{O}(3: 10: 2.5$ [v]) and were dissolved in $30 \mu \mathrm{l}$ of chloroform. Probes were applied to Polygram SIL G pre-coated TLC sheets $(20 \times 20 \mathrm{~cm}$; 0.2$\mathrm{mm}$ silica gel; Macherey-Nagel) using hexane/diethylether/formic acid (20:20:1 [v]) as the mobile phase for TAG and phospholipids. A 5- $\mu$ l aliquot of worm extract was applied to each lane to ensure that the amounts of TAGs and phospholipids were within the linear range of the standard curves. The assays were conducted in duplicate. The lipid spots were stained for $20 \mathrm{~s}$ in a dip solution containing $10 \%$ copper (II) sulfate, $8 \%$ phosphor acid, and $5 \%$ methanol. Quantification was achieved by calculating the spot intensity per area (pixel $/ \mu \mathrm{m}^{2}$ ) using AlphaEaseFC (BioRad) software. Triolein (Sigma Aldrich, Germany) and a phospholipid mixture (Sigma Aldrich, P3817) were used as standards. All of the results are presented as the mean values for the standard and diluted probes.

\section{Lipid composition analysis}

Embryos were washed three times with M9 buffer supplemented with Tween $20(1: 100,000)$ to prevent the embryos from attaching to the tubes. Defined numbers of embryos that were sorted using the COPAS Biosort system were then transferred to chloroform-rinsed Pyrex tubes and were immediately frozen at $-80{ }^{\circ} \mathrm{C}$ until being utilized for mass spectroscopy. The contents of the fatty acids triacylglyceride, phosphatidylcholine, and plasmalogen were analyzed using a high-resolution LC-MS/MS platform (Fauland et al. 2011) equipped with a Lipid Data Analyzer (Hartler et al. 2011). The results were normalized according to the contents of different fatty acid classes, the sample weight, and the protein content.

\section{Statistical analysis}

Statistical analysis was performed using Microsoft Excel (2003) and GraphPad Prism (Version 4.0) software.
Unless otherwise noted, the data represent the mean \pm SEM of at least three independent experiments. The significance of the differences was calculated using an unpaired two-tailed $t$ test. Welch's correction was applied if the variances were different. Differences were considered significant at $p<0.05(*), p<0.01(* *)$, and $p<0.001(* * *)$.

\section{Additional file}

Additional file 1: Figure S1. Similar changes of the triglyceride-tophospholipid ratio and the fatty acid composition of phosphatidylcholine (PC) in dietary restricted $L 4$ larvae (P0 generation) compared to embryos (F1 generation) obtained from dietary restricted worms. (PPTX 106 kb)

\section{Competing interests}

The authors declare that they have no competing interests.

\section{Authors' contributions}

MK carried out the DR assays, choline supplementation studies, lipid droplet analyses, and all other experiments regarding fat metabolism. MK helped to draft the manuscript. DF carried out F1 experiments regarding cold resistance and lipid droplet size. HK carried out lipodomic analyses. FD conceived of the study and participated in its design and coordination. FD drafted and finalized the manuscript. All authors read and approved the final manuscript.

\section{Acknowledgements}

We thank Astrid Reinke and Fabian Neumann for high throughput processing of plates and reagents. The study was funded by the University of Kiel and the leisure time of the authors.

\section{Author details}

${ }^{1}$ Institute of Human Nutrition and Food Science, Molecular Prevention, Christian-Albrechts University of Kiel, Heinrich-Hecht-Platz 10, 24118 Kiel, Germany. ${ }^{2}$ Institute of Genetics, TU Braunschweig, 38106 Braunschweig, Germany. ${ }^{3}$ ZMF-Center for Medical Research, University of Graz, Core Facility for Mass Spectrometry, Lipidomics and Metabolomics, A-8010 Graz, Austria. ${ }^{4}$ Omics Center Graz, A-8010 Graz, Austria.

Received: 3 December 2015 Accepted: 14 December 2015

Published online: 17 March 2016

\section{References}

Anand P, Cermelli S, Li Z, Kassan A, Bosch M, Sigua R, Huang L, Ouellette AJ, Pol A, Welte MA, Gross SP (2012) A novel role for lipid droplets in the organismal antibacterial response. eLife 1:e00003. doi:10.7554/eLife.00003

Ashrafi K, Chang FY, Watts JL, Fraser AG, Kamath RS, Ahringer J, Ruvkun G (2003) Genome-wide RNAi analysis of Caenorhabditis elegans fat regulatory genes. Nature 421(6920):268-272

Barbosa AD, Savage DB, Siniossoglou S (2015) Lipid droplet-organelle interactions: emerging roles in lipid metabolism. Curr Opin Cell Biol 35:91-97. doi:10.1016/j.ceb.2015.04.017

Barker DJP (2001) Type 2 diabetes: the thrifty phenotype_-preface. Br Med Bull 60:1-3. doi:10.1093/Bmb/60.1.1

Bartz R, Li WH, Venables B, Zehmer JK, Roth MR, Welti R, Anderson RG, Liu P, Chapman KD (2007) Lipidomics reveals that adiposomes store ether lipids and mediate phospholipid traffic. J Lipid Res 48(4):837-847

Baumeier C, Kaiser D, Heeren J, Scheja L, John C, Weise C, Eravci M, Lagerpusch M, Schulze G, Joost HG, Schwenk RW, Schurmann A (2015) Caloric restriction and intermittent fasting alter hepatic lipid droplet proteome and diacylglycerol species and prevent diabetes in NZO mice. Biochim Biophys Acta 1851(5):566-576. doi:10.1016/j.bbalip.2015.01.013

Bouwman FG, Claessens M, van Baak MA, Noben JP, Wang P, Saris WH, Mariman EC (2009) The physiologic effects of caloric restriction are reflected in the in vivo adipocyte-enriched proteome of overweight/obese subjects. J Proteome Res 8(12):5532-5540. doi:10.1021/pr900606m

Brendza KM, Haakenson W, Cahoon RE, Hicks LM, Palavalli LH, Chiapelli BJ, McLaird M, McCarter JP, Williams DJ, Hresko MC, Jez JM (2007) 
Phosphoethanolamine N-methyltransferase (PMT-1) catalyses the first reaction of a new pathway for phosphocholine biosynthesis in Caenorhabditis elegans. Biochem J 404(3):439-448. doi:10.1042/BJ20061815 Brenner S (1974) The genetics of Caenorhabditis elegans. Genetics 77(1):71-94 Burger JM, Hwangbo DS, Corby-Harris V, Promislow DE (2007) The functional costs and benefits of dietary restriction in Drosophila. Aging Cell 6(1):63-71. doi:10.1111/j.1474-9726.2006.00261.x

Cermelli S, Guo Y, Gross SP, Welte MA (2006) The lipid-droplet proteome reveals that droplets are a protein-storage depot. Curr Biol 16(18):1783-1795. doi:10.1016/j.cub.2006.07.062

Ehmke M, Luthe K, Schnabel R, Doring F (2014) S-Adenosyl methionine synthetase 1 limits fat storage in Caenorhabditis elegans. Genes Nutr 9(2): 386. doi:10.1007/s12263-014-0386-6

Fauland A, Kofeler H, Trotzmuller M, Knopf A, Hartler J, Eberl A, Chitraju C, Lankmayr E, Spener F (2011) A comprehensive method for lipid profiling by liquid chromatography-ion cyclotron resonance mass spectrometry. J Lipid Res 52(12):2314-2322. doi:10.1194/j|r.D016550

Fontana L, Partridge L (2015) Promoting health and longevity through diet: from model organisms to humans. Cell 161(1):106-118. doi:10.1016/j.cell.2015.02.020

Guo Y, Walther TC, Rao M, Stuurman N, Goshima G, Terayama K, Wong JS, Vale RD, Walter P, Farese RV (2008) Functional genomic screen reveals genes involved in lipid-droplet formation and utilization. Nature 453(7195):657-661. doi:10.1038/nature06928

Hansen M, Hsu AL, Dillin A, Kenyon C (2005) New genes tied to endocrine, metabolic, and dietary regulation of lifespan from a Caenorhabditis elegans genomic RNAi screen. PLoS Genet 1(1):119-128. doi:10.1371/journal.pgen.0010017

Hartler J, Trotzmuller M, Chitraju C, Spener F, Kofeler HC, Thallinger GG (2011) Lipid data analyzer: unattended identification and quantitation of lipids in LC-MS data. Bioinformatics 27(4):572-577. doi:10.1093/bioinformatics/btq699

Klapper M, Ehmke M, Palgunow D, Bohme M, Matthaus C, Bergner G, Dietzek B, Popp J, Doring F (2011) Fluorescence-based fixative and vital staining of lipid droplets in Caenorhabditis elegans reveal fat stores using microscopy and flow cytometry approaches. J Lipid Res 52(6):1281-1293. doi:10.1194/jlr.D011940

Krahmer N, Guo Y, Wilfling F, Hilger M, Lingrell S, Heger K, Newman HW, Schmidt-Supprian M, Vance DE, Mann M, Farese RV Jr, Walther TC (2011) Phosphatidylcholine synthesis for lipid droplet expansion is mediated by localized activation of CTP:phosphocholine cytidylyltransferase. Cell Metab 14(4):504-515. doi:10.1016/j.cmet.2011.07.013

Lee JH, Kong J, Jang JY, Han JS, Ji Y, Lee J, Kim JB (2014) Lipid droplet protein LID-1 mediates ATGL-1-dependent lipolysis during fasting in Caenorhabditis elegans. Mol Cell Biol 34(22):4165-4176. doi:10.1128/MCB.00722-14

Leermakers ET, Moreira EM, Kiefte-de Jong JC, Darweesh SK, Visser T, Voortman T, Bautista PK, Chowdhury R, Gorman D, Bramer WM, Felix JF, Franco OH (2015) Effects of choline on health across the life course: a systematic review. Nutr Rev 73(8):500-522. doi:10.1093/nutrit/nuv010

Li Y, Na K, Lee HJ, Lee EY, Paik YK (201 1) Contribution of sams-1 and pmt-1 to lipid homoeostasis in adult Caenorhabditis elegans. J Biochem 149(5):529-538. doi:10.1093/jb/mvr025

Li Z, Thiel K, Thul PJ, Beller M, Kuhnlein RP, Welte MA (2012) Lipid droplets control the maternal histone supply of Drosophila embryos. Curr Biol 22(22): 2104-2113. doi:10.1016/j.cub.2012.09.018

Matlib MA, Frenkel EP, Mukherjee A, Henslee J, Srere PA (1979) Enzymatic properties of mitochondria isolated from normal and vitamin B12-deficient rats. Arch Biochem Biophys 197(2):388-395

Matyash V, Liebisch G, Kurzchalia TV, Shevchenko A, Schwudke D (2008) Lipid extraction by methyl-tert-butyl ether for high-throughput lipidomics. J Lipid Res 49(5):1137-1146. doi:10.1194/Jlr.D700041-JLR200

Miersch C, Doring F (2013) Sex differences in body composition, fat storage, and gene expression profile in Caenorhabditis elegans in response to dietary restriction. Physiol Genomics 45(13):539-551. doi:10.1152/physiolgenomics.00007.2013

Narbonne P, Roy R (2009) Caenorhabditis elegans dauers need LKB1/AMPK to ration lipid reserves and ensure long-term survival. Nature 457(7226):210-214. doi:10.1038/nature07536

Nishino N, Tamori Y, Tateya S, Kawaguchi T, Shibakusa T, Mizunoya W, Inoue K, Kitazawa R, Kitazawa S, Matsuki Y, Hiramatsu R, Masubuchi S, Omachi A, Kimura K, Saito M, Amo T, Ohta S, Yamaguchi T, Osumi T, Cheng J, Fujimoto T, Nakao H, Nakao K, Aiba A, Okamura H, Fushiki T, Kasuga M (2008) FSP27 contributes to efficient energy storage in murine white adipocytes by promoting the formation of unilocular lipid droplets. J Clin Invest 118(8): 2808-2821. doi:10.1172/JCl34090
Palavalli LH, Brendza KM, Haakenson W, Cahoon RE, McLaird M, Hicks LM, McCarter JP, Williams DJ, Hresko MC, Jez JM (2006) Defining the role of phosphomethylethanolamine $\mathrm{N}$-methyltransferase from Caenorhabditis elegans in phosphocholine biosynthesis by biochemical and kinetic analysis. Biochemistry 45(19):6056-6065. doi:10.1021/bi060199d

Palgunow D, Klapper M, Doring F (2012) Dietary restriction during development enlarges intestinal and hypodermal lipid droplets in Caenorhabditis elegans. PLoS One 7(11):e46198. doi:10.1371/journal.pone.0046198

Rinella ME, Green RM (2004) The methionine-choline deficient dietary model of steatohepatitis does not exhibit insulin resistance. J Hepatol 40(1):47-51

Szymanski KM, Binns D, Bartz R, Grishin NV, Li WP, Agarwal AK, Garg A, Anderson RG, Goodman JM (2007) The lipodystrophy protein seipin is found at endoplasmic reticulum lipid droplet junctions and is important for droplet morphology. Proc Natl Acad Sci USA 104(52):20890-20895

Walker AK, Jacobs RL, Watts JL, Rottiers V, Jiang K, Finnegan DM, Shioda T, Hansen M, Yang F, Niebergall $L$, Vance DE, Tzoneva M, Hart AC, Naar AM (2011) A conserved SREBP-1/phosphatidylcholine feedback circuit regulates lipogenesis in metazoans. Cell 147(4):840-852. doi:10.1016/j.cell.2011.09.045

Welte MA (2015) Expanding roles for lipid droplets. Curr Biol 25(11):R470-R481. doi:10.1016/j.cub.2015.04.004

Zeisel SH, Da Costa KA, Franklin PD, Alexander EA, Lamont JT, Sheard NF, Beiser A (1991) Choline, an essential nutrient for humans. FASEB J 5(7):2093-2098

Zhang SO, Box AC, Xu N, Le Men J, Yu J, Guo F, Trimble R, Mak HY (2010a) Genetic and dietary regulation of lipid droplet expansion in Caenorhabditis elegans. Proc Natl Acad Sci U S A 107(10):4640-4645. doi:10.1073/pnas. 0912308107

Zhang SO, Trimble R, Guo F, Mak HY (2010b) Lipid droplets as ubiquitous fat storage organelles in C. elegans. BMC Cell Biol 11:96. doi:10.1186/1471-2121-11-96

\section{Submit your next manuscript to BioMed Central and we will help you at every step:}

- We accept pre-submission inquiries

- Our selector tool helps you to find the most relevant journal

- We provide round the clock customer support

- Convenient online submission

- Thorough peer review

- Inclusion in PubMed and all major indexing services

- Maximum visibility for your research

Submit your manuscript at www.biomedcentral.com/submit

C) Biomed Central 